\title{
Utilisation de pratiques agroécologiques et performances de la petite agriculture familiale : le cas de la Guadeloupe
}

\author{
Camille Rasse ${ }^{1}$, Nadine Andrieu ${ }^{2,3,4, *}$, Jean-Louis Diman ${ }^{1}$, Audrey Fanchone ${ }^{5}$ et Eduardo Chia ${ }^{4}$ \\ ${ }^{1}$ Inra, UE 0805 PEYI, centre Antilles-Guyane, 97170 Petit-Bourg, Guadeloupe, France \\ 2 Cirad, UMR Innovation, Cali, Colombie \\ 3 CIAT, AA 6713, Cali, Colombie \\ ${ }^{4}$ Innovation, université de Montpellier, Montpellier, France \\ 5 Inra, UR 143 Zootechnie, 97170 Petit-Bourg, Guadeloupe, France
}

\begin{abstract}
Résumé - L'analyse et la quantification des performances de la petite agriculture familiale (PAF) constituent un enjeu au regard de la transition agroécologique. Cet article utilise une méthode combinant enquêtes auprès d'agriculteurs de la PAF de Guadeloupe, typologie et évaluation, à l'échelle de l'exploitation, des avantages qu'ils tirent de pratiques agroécologiques en termes de production de biomasse, de couverture des besoins caloriques de la famille, d'efficience azotée, d'économie d'intrants ou de production de valeur ajoutée. Il montre que la PAF est diverse et hybride usage de pratiques agroécologiques et d'intrants de synthèse. En fonction du nombre de pratiques agroécologiques mises en œuvre et de l'orientation de la production, on observe des synergies entre le nombre d'espèces domestiquées, la diminution des charges intermédiaires et la couverture des besoins de la famille. Des tensions ont en revanche été observées entre ces indicateurs et la valeur ajoutée brute commerciale. Des synergies sont néanmoins possibles entre la majorité des indicateurs lorsque le producteur intègre des espèces à haute valeur ajoutée, ce qui permet d'identifier des pistes pour favoriser la transition agroécologique de ces systèmes.
\end{abstract}

Mots clés : systèmes mixtes de polyculture-élevage / Caraïbes / agriculture tropicale

\begin{abstract}
Use of agroecological practices and performances of small family farming: the case of Guadeloupe. The analysis and quantification of the performance of small family agriculture (SFA) is an issue with regard to the agroecological transition. This article uses a methodology combining surveys with SFA farmers in Guadeloupe, typology and assessment, at the farm level, of the benefits associated with the use of agroecological practices in terms of biomass production, supply of calories for the family, nitrogen efficiency, input saving, or production of added value. It shows that SFA in Guadeloupe is diverse and uses both agroecological practices and chemical inputs. According to the number of agroecological practices they use and to the orientation of the production, synergies were observed between domestic specific diversity, input saving, and caloric self-sufficiency of the family; trade-offs were observed between these indicators and gross value added. However, synergies are possible between the majority of the indicators when farmers use species with high economic value, which makes it possible to identify ways for agroecological transition.
\end{abstract}

Keywords: mixed crop-livestock systems / The Caribbean / tropical agriculture

\section{Introduction}

L'agriculture familiale (Sourisseau, 2015) représente la majorité des exploitations au niveau mondial et joue un rôle clé

\footnotetext{
*Auteur de correspondance : nadine.andrieu@cirad.fr
}

pour la sécurité alimentaire (Graeub et al., 2016). Une des caractéristiques de cette agriculture est sa résilience, du fait de sa capacité à mobiliser des réseaux sociaux, couplée ou non à celle de pouvoir mobiliser les services écosystémiques via des pratiques agroécologiques (Darnhofer et al., 2016). Une frange de cette agriculture familiale relève de la petite agriculture, généralement lorsque les surfaces cultivées sont inférieures à 


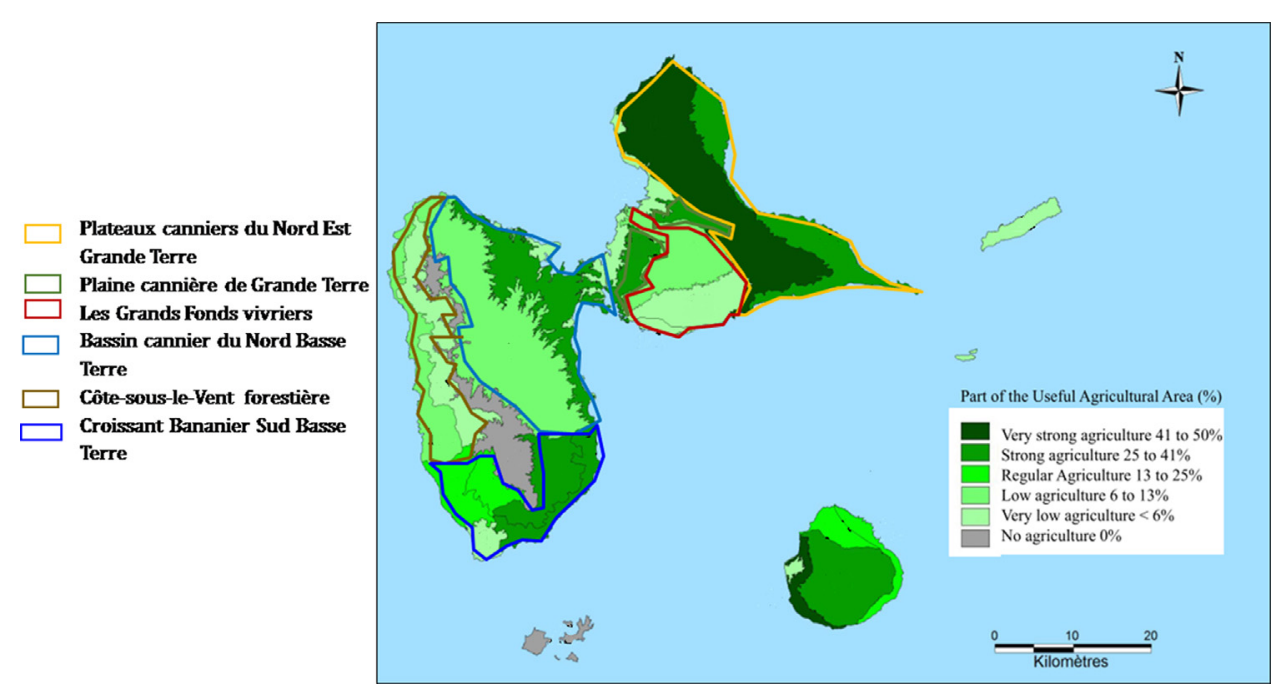

Fig. 1. Principales zones agricoles de la Guadeloupe. Source : fond de carte (Mantran et al., 2016); zones agricoles (Semjen, 2017).

Fig. 1. Main agricultural zones of Guadeloupe.

2 hectares (Lowder et al., 2016). L'analyse et la quantification des performances de la petite agriculture familiale (PAF) constituent un enjeu au regard de la transition agroécologique.

En Guadeloupe, il existe une PAF peu représentée au sein des circuits conventionnels (accès aux structures de conseil et de développement et aux aides) et peu visible dans les statistiques agricoles, en particulier s'agissant de la production agricole finale (Bezombes et al., 2015). Les agriculteurs ne déclarent pas, ou partiellement, leur volume d'activité, mais auraient un poids non négligeable dans la fourniture du marché alimentaire local. Ce qualificatif de «PAF» ne se base pas de façon stricte sur un critère de surface, mais à la fois sur son caractère informel et sur les revenus générés (Inra, 2015). Si cette agriculture apparait dans les descriptions qui sont faites de l'agriculture guadeloupéenne (Stark et al., 2016), son caractère multiforme, la nature des pratiques agroécologiques que mettent en ouvre (ou non) les agriculteurs qui la composent, et les performances associées restent encore peu étudiées.

Nous présentons les résultats d'une recherche visant à explorer en quoi les pratiques agroécologiques mobilisées par la PAF contribuent aux performances des exploitations agricoles. Après avoir présenté la méthode, nous décrivons la diversité des systèmes de production rencontrés et leurs performances avant de discuter de leur contribution potentielle à l'agroécologie.

\section{Matériel et méthodes}

\subsection{Zone d'étude}

L'étude a été menée en Guadeloupe continentale composée de la Basse-Terre à l'ouest $\left(848 \mathrm{~km}^{2}\right)$ et de la Grande-Terre à l'est $\left(590 \mathrm{~km}^{2}\right)$.

Le climat est caractérisé par deux saisons principales : la saison sèche (de janvier à avril) et la saison des pluies (de juillet à octobre). Elles sont séparées par deux périodes de transition. Le cycle annuel des températures moyennes est marqué par une faible amplitude : 2 à $3{ }^{\circ} \mathrm{C}$ pour les minimales et 3 à $4{ }^{\circ} \mathrm{C}$ pour les maximales (Météo France, 2017).
La présence et la répartition des reliefs entre Basse-Terre et Grande-Terre jouent un rôle majeur dans le climat de ces îles et conditionnent la répartition des précipitations. Le plateau calcaire de la Grande-Terre, avec une pluviométrie moyenne de $2300 \mathrm{~mm}$ par an, connaît régulièrement des périodes de sécheresse. Tandis qu'en Basse-Terre, le relief, perpendiculaire au flux des alizés et aux vents venus de l'Est, régule le régime des pluies qui va de $900 \mathrm{~mm}$ par an en Côte-sous-le-vent à $3800 \mathrm{~mm}$ par an sur la Côte-au-vent.

Différentes zones agricoles ont été identifiées en fonction des systèmes de culture dominants (Fig. 1). L'activité agricole couvre plus du tiers de la superficie de l'archipel et est présente partout à l'exclusion des crêtes montagneuses et du parc national. Les canniers se trouvent majoritairement sur les plateaux et les plaines de Grande-Terre et du nord de BasseTerre. Les Grands-Fonds permettent les cultures de tubercules et racines. Le sud de Basse-Terre et la Côte-au-vent portent le nom de «croissant bananier» et la Côte-sous-le-vent est une zone forestière.

\subsection{Démarche méthodologique}

Les pratiques agroécologiques appliquent un certain nombre de principes tels que le recyclage de la biomasse, le renforcement de la biodiversité fonctionnelle (plantes antagonistes, ennemis naturels...), la conservation et la régénération des eaux, des sols et de l'agrobiodiversité pour limiter les pertes de ressources (énergie, eau, nutriments), la diversification des espèces et des ressources génétiques, les interactions et les synergies entre les composantes de l'agrobiodiversité (Altieri, 2002). Ce sont, en d'autres termes, des pratiques qui favorisent la provision de services écosystémiques d'approvisionnement, de support ou de régulation (Altieri, 1999). Pour analyser en quoi les pratiques agroécologiques mobilisées par la PAF contribuent aux performances des exploitations agricoles, nous avons fait le choix d'évaluer deux dimensions : les caractéristiques agroécologiques des exploitations et les avantages qu'en tirent les agriculteurs. Ces avantages correspondent à ce que les agriculteurs créent ou font dériver 
a)

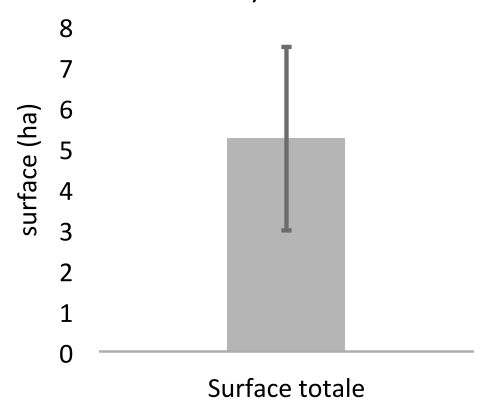

b)

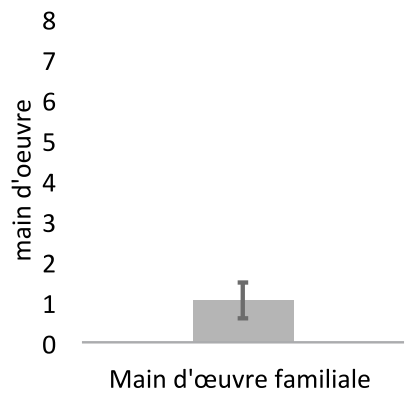

c)

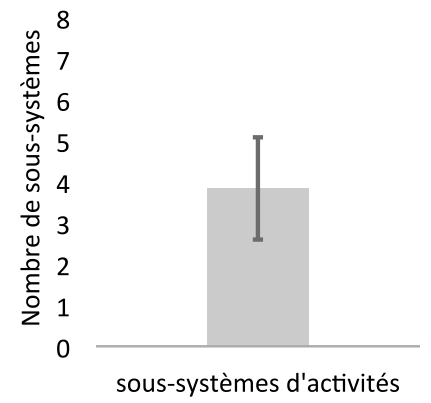

Fig. 2. Moyenne et écart-type des surfaces (a), nombre d'actifs familiaux (b) et nombre de sous-systèmes de production (c) pour les 20 exploitations étudiées.

Fig. 2. Mean and standard deviation of the surface areas (a), number of family assets (b) and number of production subsystems (c) for the 20 surveyed farms.

des services écosystémiques via les pratiques agroécologiques (CICES, 2013). Il peut s'agir par exemple de l'augmentation de la production ou de la réduction de l'usage des intrants de synthèse.

Pour évaluer ces avantages, nous nous sommes inspirés de travaux de Sempore et al. (2015), qui montrent que les outils de calcul du bilan de l'exploitation, comparés à ceux simulant les règles de décision de l'agriculteur ou d'optimisation, permettent aux agriculteurs et aux techniciens les ayant utilisés de représenter de façon plus flexible le fonctionnement de l'exploitation.

Pour développer cet outil, nous avons :

- élaboré une typologie de la PAF afin de caractériser sa diversité ;

- construit un outil d'analyse des deux dimensions: les caractéristiques agroécologiques de l'exploitation et les avantages qu'en tirent les agriculteurs calculés à travers des bilans entre offre et demande des ressources à l'échelle de l'exploitation;

- recueilli les données auprès d'exploitations représentatives des types;

- simulé les performances de ces différents types.

\subsubsection{Typologie}

Nous avons choisi les producteurs à interroger au sein d'une base de données de 92 agriculteurs, constituée dans le cadre d'un projet de recherche antérieur financé par l'ANR (projet 12-AGRO-0009-Gaia-Trop 2014-2017). Cette base a été construite selon la méthode d'échantillonnage par boule de neige (Laws et al., 2013) au sein des différentes zones agricoles de Guadeloupe (Fig. 1).

Pour choisir les producteurs de notre étude, nous avons considéré les critères suivants en nous basant sur une caractérisation préexistante de la PAF proposée par 70 acteurs du territoire lors d'un atelier antérieur à notre recherche (Inra, 2015):

- taille de l'exploitation inférieure ou égale à 10 hectares ;

- au moins deux sous-systèmes de production (cultures associées ou jardin considérés comme un sous-système de production);

- au moins $50 \%$ de la main-d'œuvre est familiale.
Vingt producteurs correspondaient à ces trois critères (Fig. 2). Nous avons ensuite récolté par entretien semi-directif les données suivantes :

- sociales (âge, niveau scolaire, famille, origine des savoirs, lien avec les acteurs agricoles);

- structurelles (ateliers de culture et d'élevage, matériel, main-d'œuvre...);

- agronomiques (pratiques conventionnelles et agroécologiques pour les différents systèmes de culture et d'élevage);

- économiques (type de spéculation, commercialisation);

- historique et vision pour l'avenir.

Pour élaborer notre typologie, nous avons regroupé les exploitations présentant les mêmes systèmes de culture ou d'élevage principaux (en termes de stratégie et de surface).

\subsubsection{Construction d'un outil d'évaluation des performances de la petite agriculture familiale}

\subsubsection{Choix des indicateurs}

Pour faciliter le recueil et l'analyse des données, nous avons retenu un nombre limité d'indicateurs ad hoc. Nous avons considéré 8 indicateurs pour les caractéristiques agroécologiques et avantages évalués. Ils sont décrits dans la section cidessous, ainsi que leur mode de calcul, les variables d'entrées utilisées (en italique) et les paramètres fixes (en gras).

La première dimension (caractéristiques agroécologiques) comporte deux indicateurs :

- le premier décrit la richesse spécifique domestique qui correspond à la biodiversité planifiée par l'agriculteur (Altieri, 1999) [BD]. Celle-ci est composée des espèces végétales et animales sélectionnées par les agriculteurs et joue un rôle dans la provision de services écosystémiques : $B D=$ (nombre espèces animales + nombre espèces végétales domestiquées) / surface totale de l'exploitation;

- le second est le nombre de pratiques agroécologiques par hectare [nbr PAE]. Il permet d'évaluer l'importance de ces pratiques dans la stratégie de production:

$\mathrm{Nbr} \operatorname{PAE}=(\Sigma$ pratiques agroécologiques atelier de culture $+\Sigma$ pratiques agroécologiques atelier d'élevage) / surface totale de l'exploitation. 
La seconde dimension (avantages que tirent les agriculteurs) comporte six indicateurs :

- le rendement du système de production en unité de poids par unité de surface [Rdmt]. Ilévalue la capacité de l'exploitation à produire une quantité de produits issus des ateliers d'élevage et de culture (grains, fruits et fourrages) :

$\mathrm{Rdmt}=(\Sigma$ production atelier de culture $+\Sigma$ effectif atelier élevage $\times$ poids moyen animal)/surface totale de l'exploitation;

- le taux de recouvrement des besoins caloriques du ménage par les produits de l'exploitation [rec Bes Fam] (Burgess, 2005). Il évalue la capacité du système de production à couvrir les besoins alimentaires du ménage:

rec Bes Fam $=\Sigma$ besoin calorique par classe d'âge $\times$ effectif de chaque classe d'âge / ( $\Sigma$ (produit cultivé autoconsommé $\times$ valeur calorique produit) $+\Sigma$ (produit animal autoconsommé $\times$ valeur calorique produit) $) \times 100$;

- l'efficience d'utilisation de l'azote [Eff N], qui prend en compte l'ensemble des sorties (en kilogrammes azotés de viande et/ou de culture vendues) et des apports azotés de l'exploitation (sous-produits agricoles et compléments pour les différents ateliers d'animaux, engrais minéraux ou organiques pour les ateliers de cultures) (Godinot, 2014): Eff $\mathrm{N}=(\Sigma$ produit cultivé vendu $\times$ taux de matière sèche $\times$ valeur azotée par $\mathrm{kg}$ de culture + produit élevage vendu $\times$ valeur azotée animal $) /(\Sigma$ quantité intrant culture $\times$ valeur azotée intrant culture + quantité intrant élevage $\times$ valeur azotée intrant élevage).

Pour les cultures associées, ce sont les sorties et les apports du système de cultures associées qui sont considérés :

- la limitation des charges intermédiaires liées à l'achat de produits de synthèse [Lim $\mathrm{CI}]$. L'indicateur évalue la capacité de l'exploitation à limiter les frais liés aux intrants de synthèse des ateliers de cultures et d'élevage. Il s'agit donc de l'inverse des charges par hectare:

Lim $\mathrm{CI}=$ surface totale de l'exploitation/ $(\Sigma$ (coûts engrais minéraux + coûts herbicides + coûts pesticides $)+\Sigma$ (coûts vermifuge + anti-tique + autres produits));

- la valeur ajoutée brute «commerciale» par hectare [VAB/ ha] et la valeur ajoutée brute par homme jour [VAB/hj]. Ces deux indicateurs permettent de mesurer la richesse «commerciale» produite par unité de surface et par journée de travail investie dans le système de production: $\mathrm{VAB} / \mathrm{ha}=\Sigma$ (produit cultivé vendu $\times$ prix de vente-charges intermédiaires cultures)/durée de la rotation+ (produit élevage vendu $\times$ prix de vente-charges intermédiaires élevage))/SAU totale de l'exploitation agricole; $\mathrm{VAB} / \mathrm{hj}=(V A B / h a) /$ total de jours travaillés par hectare et par actif.

\subsubsection{Pas de temps}

L'outil considère les 12 mois de l'année (de décembre année $n$ - 1 à novembre de l'année $n$ ), afin de prendre en compte les variations de rendement et de prix.

\subsubsection{Structure de l'outil}

L'outil est constitué de sept modules représentant l'exploitation agricole (Fig. 3). Il existe pour chacun d'entre eux une feuille de calcul Excel comportant:
- des variables d'entrée, renseignées par l'utilisateur pour les différentes cultures et types d'animaux (surfaces cultivées, effectifs d'animaux, produits vendus ou autoconsommés, quantités d'intrants utilisées par atelier de production). Les associations de culture sont à renseigner sur une unité d'espace donnée, sans faire de différences entre les espèces qui la composent;

- des paramètres fixes issus de la littérature, tels que la valeur calorique des produits autoconsommés ou la composition azotée des différents types de fumure;

- les 8 indicateurs utilisés pour analyser les caractéristiques agroécologiques et avantages à l'échelle de l'exploitation (Fig. 3).

\subsubsection{Simulation des performances d'exploitations types}

Pour cette étape, nous avons recueilli les données d'entrées nécessaires aux calculs lors d'enquêtes directives avec deux agriculteurs pour chacun des types identifiés lors de l'étape précédente. Les deux agriculteurs interrogés au sein de chaque type se différenciaient par leur nombre de pratiques agroécologiques.

La valeur calculée de chaque indicateur a été notée de 1 (valeur la plus faible pour les huit exploitations considérées) à 8 (valeur la plus élevée), de façon à disposer d'une échelle commune pour représenter l'ensemble des indicateurs sur un diagramme en radar.

\section{Résultats}

\subsection{Pratiques agroécologiques et types de systèmes de production identifiés}

Dix principales pratiques agroécologiques ont été identifiées, toutes exploitations confondues (Fig. 4). Elles visaient généralement à favoriser l'infiltration de l'eau (couvert végétal et paillage) et à limiter les charges intermédiaires, en particulier l'usage de fertilisants minéraux (jachère, légumineuse, cendre, recyclage des fèces, rotation, purin/décoction), de produits phytosanitaires (étouffement des adventices, association de cultures, eau de mer, algue) ou encore de compléments alimentaires (alimentation des animaux avec des co-produits de cultures intra- et/ou extra-exploitation).

Nous avons également identifié 5 types d'exploitations en fonction des sous-systèmes de production dominants (Tab. 1) :

- maraîchage et culture pérenne ou de sécurité à forte valeur ajoutée ;

- verger et élevage;

- verger et maraîchage;

- élevage et canne à sucre ;

- élevage et jardin.

La diversité des pratiques agroécologiques rencontrées au sein d'une même exploitation varie en fonction du type. Ainsi, les types avec un atelier principal en verger utilisent peu de pratiques agroécologiques, du fait d'une conduite de l'arbre (café, manguier, avocatier) qui nécessite peu d'interventions. Dans les systèmes de production en élevage et canne à sucre, l'accès aux intrants de synthèse est facilité par les coopératives; les pratiques agroécologiques sont alors quasi 
Avantages (Rendement, efficience de l'utilisation de l'azote, VAB, recouvrement des besoins caloriques du ménage, limitation des charges)

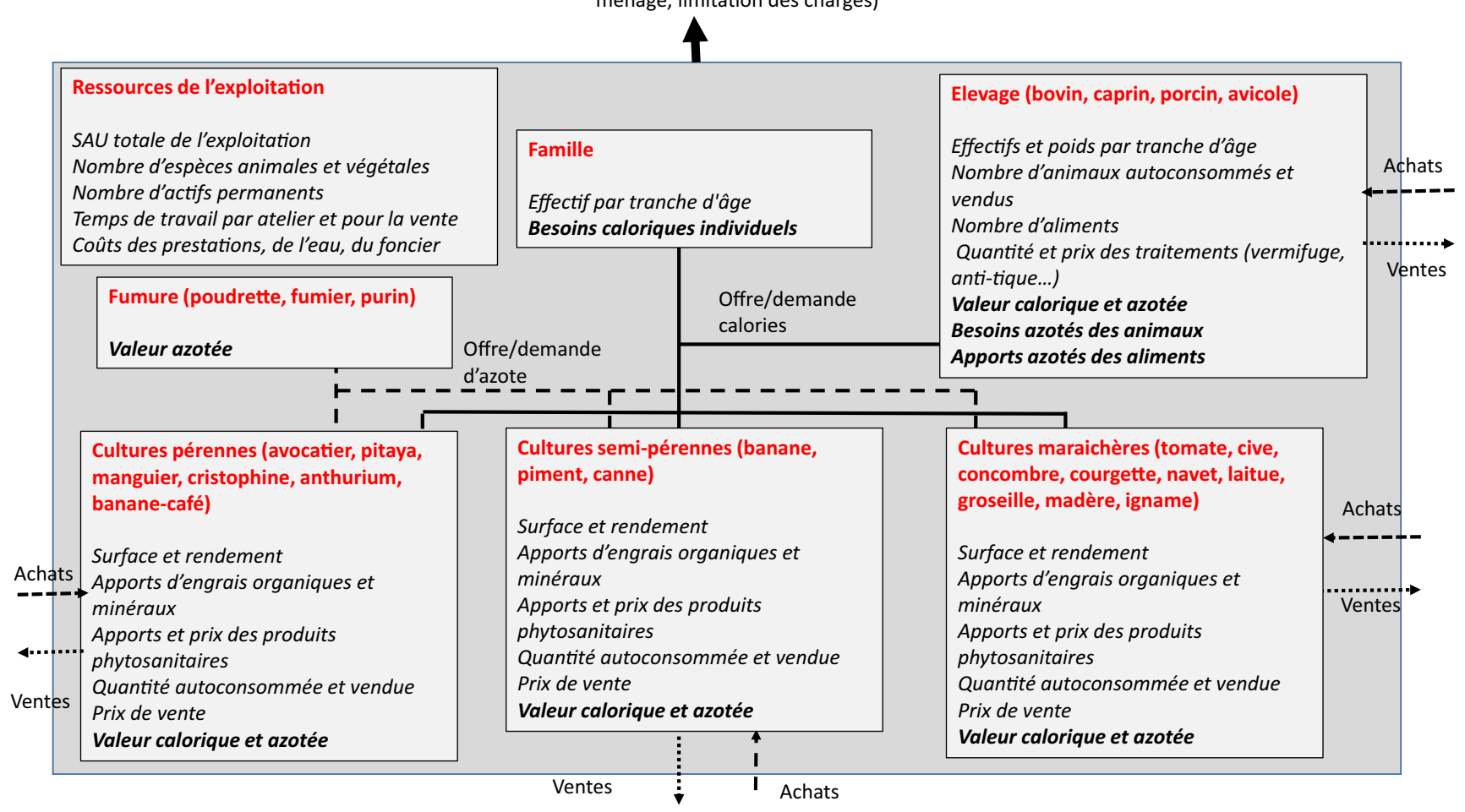

Fig. 3. Structure de l'outil. Les noms des différents modules sont en rouge; les flèches en pointillés représentent les flux (de fumure, d'aliments) entre les modules; les paramètres sont en gras et en italiques; les données d'entrée sont en italiques.

Fig. 3. Structure of the tool. The names of the various modules are in red; the dashed arrows represent the flows (of manure, food) between the modules; the parameters are in bold and italics; the input data are in italics.

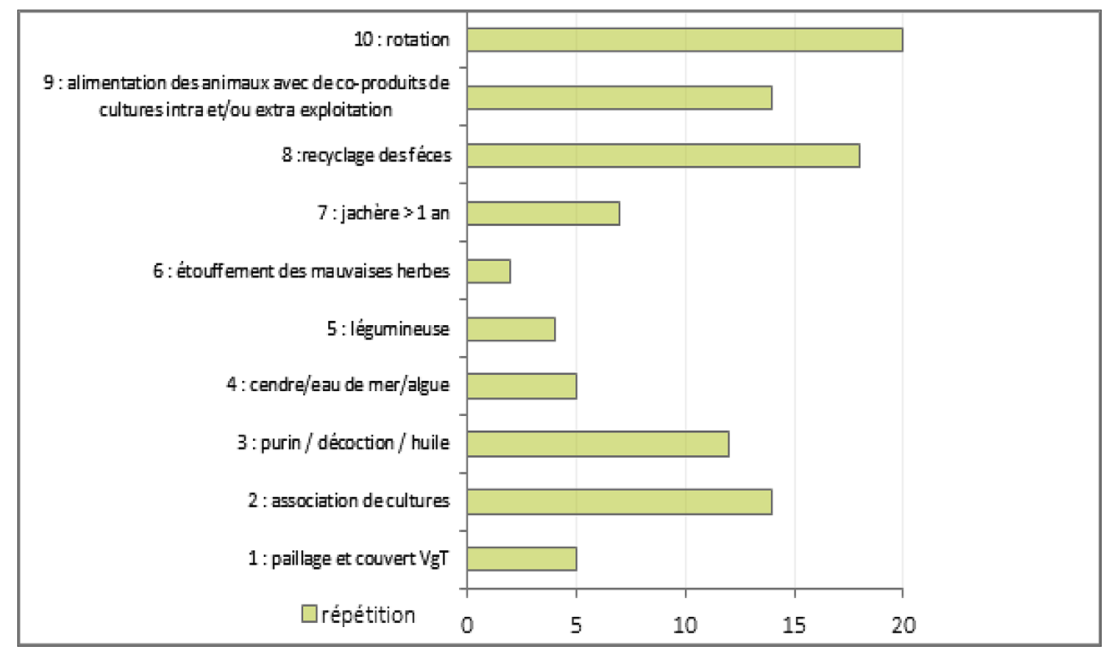

Fig. 4. Pratiques agroécologiques mentionnées par les producteurs interrogés.

Fig. 4. Agroecological pratices mentioned by the surveyed farmers.

absentes. Dans les exploitations en élevage et jardin, la diversité de pratiques agroécologiques est forte, avec des pratiques telles que : le paillage, les associations de cultures, le recyclage des fèces, les rotations. Ce choix peut s'expliquer par l'orientation de la production pour l'autoconsommation, mais aussi du fait d'une plus grande prise de risque rendue possible par la double activité chez ces agriculteurs. Cependant, cette diversité de pratiques agroécologiques est aussi en lien avec les contraintes physiques de l'exploitation puisque, au sein d'un même type, ces pratiques sont plus variées pour les exploitations ayant les surfaces les plus limitées ou les plus pentues. 
C. Rasse et al. : Cah. Agric. 2018, 27, 55002

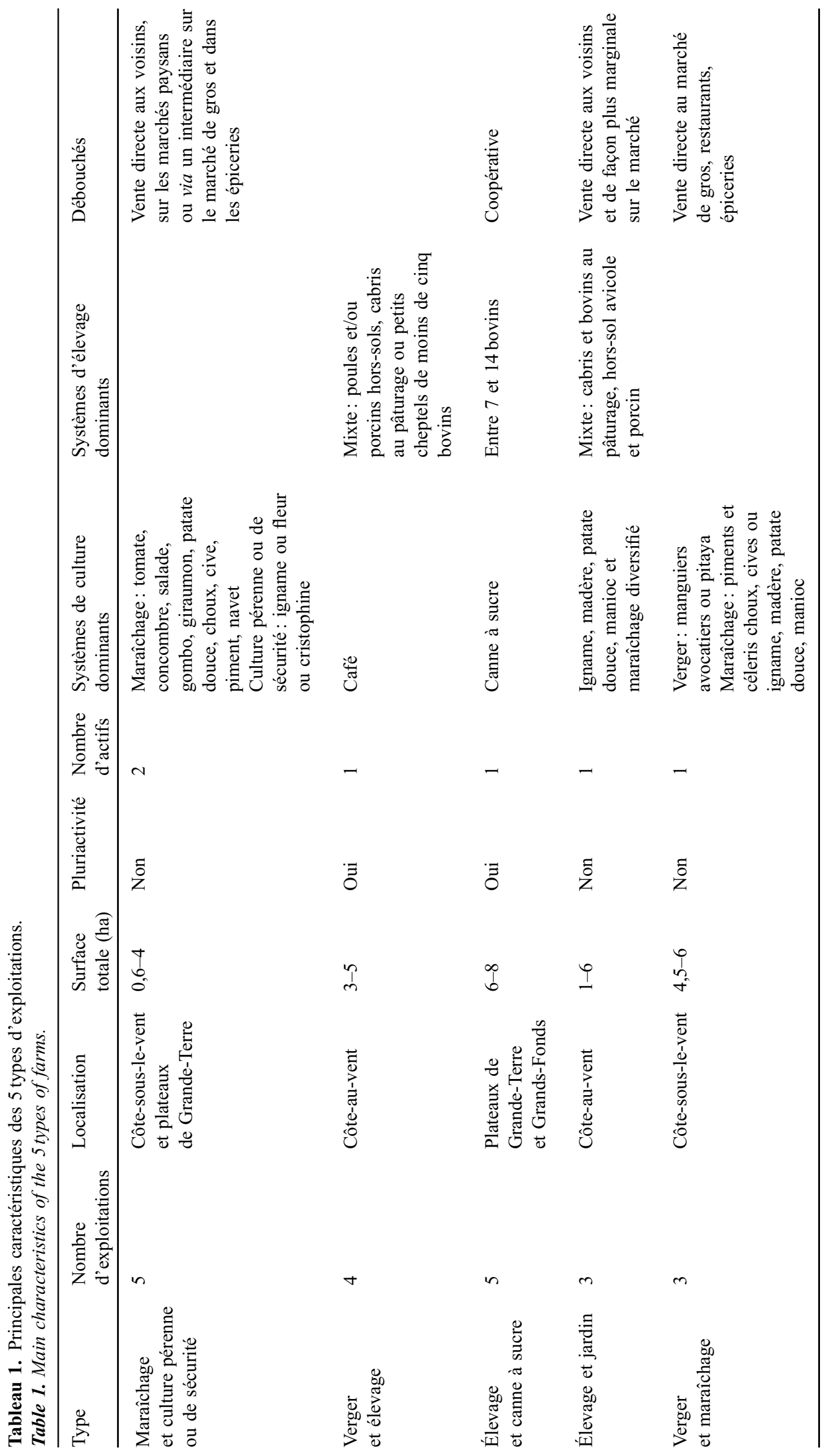

Page 6 de 10 
Tableau 2. Caractéristiques des 8 cas d'études au sein de la petite agriculture familiale.

Table 2. Main characteristics of the 8 farms.

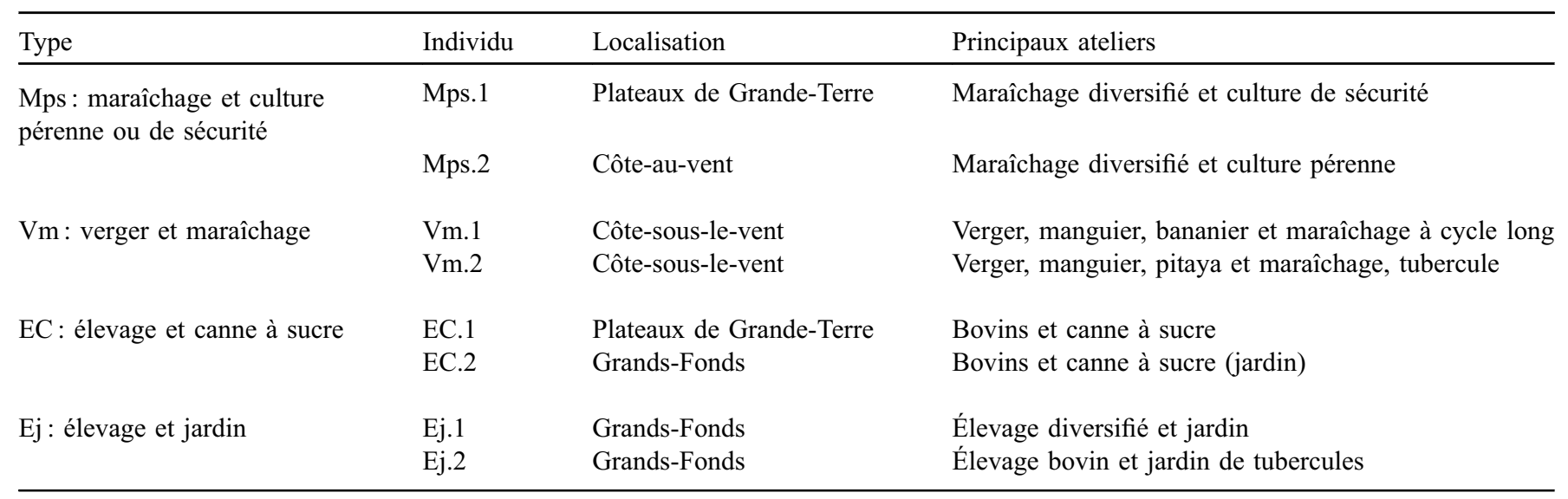
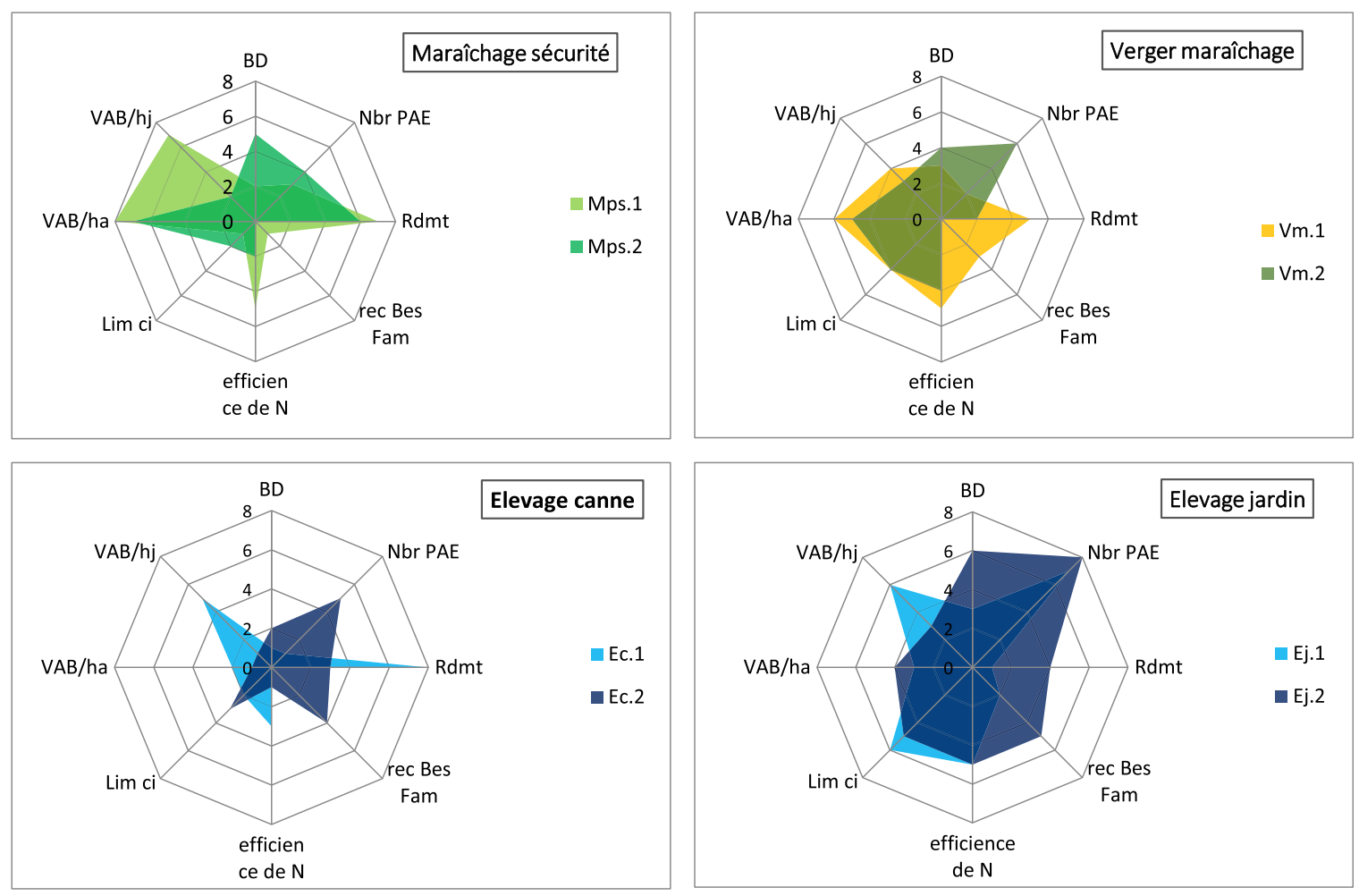

BD : biodiversité spécifique ; Nbr PAE : nombre de pratiques agroécologiques

Rdmt : rendement en Kcal; rec Bes Fam : recouvrement des besoins de la famille; Efficience de $\mathbf{N}$ : efficience de l'azote; Lim ci : limitation des charges intermédiaires; VAB/ha : valeur ajoutée brute commerciale par hectare; $\mathbf{V A B} / \mathbf{H j}$ : valeur ajoutée brute commerciale par homme jour

Fig. 5. Performances simulées des exploitations (se référer au Tab. 2 pour une description des individus).

Fig. 5. Simulated performances of farms (refer to Tab. 2 for a description of individuals).

\subsection{Performances de la petite agriculture familiale}

Pour cette section, nous nous sommes appuyés sur 4 des 5 types identifiés (Tab. 2). Le type verger et élevage est en reconversion; le système n'étant pas en production, il n'a pas été possible de collecter de données quantitatives nécessaires à la simulation.
On constate qu'aucun des types analysés n'obtient de bonnes performances sur l'ensemble des indicateurs considérés (Fig. 5). Par exemple, les types avec un atelier de maraîchage dominant (types verger et maraîchage ou maraîchage et culture pérenne ou de sécurité à forte valeur ajoutée) obtiennent les meilleurs scores en termes de valeur ajoutée brute/ha. Ces scores correspondent à des valeurs 
simulées par l'outil comprises entre $6500 € /$ ha et $2300 € /$ ha, tandis qu'elles oscillent entre $700 € /$ ha et $100 € /$ ha pour les autres types. Le type élevage et jardin obtient, quant à lui, les meilleurs scores en termes de limitation des charges intermédiaires ou d'efficience azotée. En effet, les ressources de l'exploitation sont recyclées au sein du système de production, les intrants extérieurs sont limités et la production est autoconsommée. Dans ces exploitations, les besoins caloriques simulés des ménages sont couverts à près de $90 \%$ par les produits de l'exploitation.

En règle générale, on constate que, indépendamment du type, les exploitations combinant une plus grande diversité de pratiques agroécologiques (couleurs plus foncées sur chacun des graphiques de la Fig. 5) obtiennent des scores plus élevés en termes de biodiversité et de diminution des charges intermédiaires. On peut donc dire qu'il y a des synergies entre ces indicateurs. En revanche, dans ce cas de figure, les scores obtenus sont plus faibles en termes de valeur ajoutée brute commerciale par hectare, mais surtout par jour de travail, du fait d'un rendement généralement plus faible pour ces exploitations et/ou d'un investissement plus important en travail. Ces deux indicateurs sont donc en tension avec ceux relatifs aux caractéristiques agroécologiques. Pour les types élevage et canne ainsi qu'élevage et jardin, les exploitations combinant une plus grande diversité de pratiques agroécologiques obtiennent les meilleurs scores en termes de couverture des besoins caloriques du ménage. Pour les types maraîchages et culture pérenne ou de sécurité, ainsi que verger et maraîchage, l'introduction de davantage de pratiques agroécologiques ne se traduit pas par une amélioration de la couverture des besoins caloriques du ménage, du fait d'une orientation privilégiée de la production vers le marché.

Nous n'avons pas pu identifier de lien entre pratiques agroécologiques et efficience azotée, car certains des producteurs ayant introduit davantage de pratiques agroécologiques (en particulier dans le cas des cultures associées) ont pu limiter les quantités de pesticides, mais pas les quantités d'engrais minéraux.

\section{Discussion}

\subsection{Quelles voies pour la transition agroécologique de la petite agriculture familiale?}

Cette étude a mis en évidence la diversité structurelle de la PAF, l'hybridation qu'elle fait entre pratiques conventionnelles et agroécologiques et les synergies associées entre diversité spécifique domestique, limitation des charges intermédiaires et couverture des besoins caloriques du ménage, souvent en tension avec la valeur ajoutée brute commerciale, qu'elle soit par hectare ou par homme jour. Ces tensions et synergies entre les avantages tirés des services écosystémiques ont été mises en évidence par d'autres chercheurs (Power, 2010) et sont inhérentes aux systèmes familiaux où une diversité d'objectifs sont recherchés. Seul un des cinq types est en voie de substitution totale des pratiques conventionnelles par les pratiques agroécologiques. Il s'agit du type élevage et jardin, qui est celui mobilisant la plus grande diversité de pratiques agroécologiques. Il pourrait alors correspondre à une transition vers une «forme forte» de modernisation écologique de l'agriculture, où l'on cherche à substituer les intrants de synthèse par les services écosystémiques, par opposition à une « forme faible» qui est définie comme une simple recherche d'efficience des intrants (Duru et al., 2014).

Plusieurs pistes peuvent ainsi être proposées pour favoriser les synergies entre valeur ajoutée brute commerciale et les autres avantages tirés des services écosystémiques, et, in fine, pour favoriser la transition agroécologique de la PAF. Les cas de synergie avec la valeur ajoutée brute commerciale par hectare concernent les systèmes ayant introduit comme atelier principal du maraîchage avec des cultures à forte valeur ajoutée, comme des cultures de contre-saison. Néanmoins, ces systèmes sont exigeants en main-d'œuvre et n'ont été observés que dans les exploitations ayant au moins deux actifs à temps plein.

Une autre piste pour améliorer les synergies entre valeur ajoutée brute et autres avantages tirés des services écosystémiques, mentionnée par 11 des 20 agriculteurs interrogés lors de l'étape 1 , et par 3 des 8 agriculteurs interrogés lors de l'étape 3 , est celle de l'introduction de vergers permettant de limiter l'usage d'intrants compte tenu du faible entretien nécessaire dans des ménages souvent pluriactifs. Mais des pistes sont également à rechercher hors de l'exploitation, en améliorant en particulier les débouchés des productions agricoles, qui existent et sont multiples, mais ne sont pas organisés.

\subsection{Intérêts et limites de la méthode}

La démarche suivie s'appuie sur la combinaison de différentes méthodes: enquêtes compréhensives et directives, construction d'une typologie, couplées à la construction d'un outil d'évaluation des performances de la PAF. Le type d'outil utilisé calcule différents bilans à l'échelle de l'exploitation et s'apparente à ceux proposés par plusieurs auteurs pour analyser et évaluer le fonctionnement des exploitations (Sempore et al., 2015; Le Gal et al., 2013). Néanmoins, les indicateurs de caractérisation et bilans calculés ici (par exemple, biodiversité planifiée par hectare, bilan calorique, efficience azotée) visent à analyser de façon spécifique le lien entre pratiques agroécologiques et avantages que peuvent en tirer les agriculteurs. La prise en compte d'indicateurs variés permet en outre de tenir compte d'une PAF multiforme. L'utilisation de l'outil couplée à la typologie a permis d'affiner les connaissances existantes (Semjen, 2015; Stark et al., 2016) sur des agriculteurs peu représentés au sein des circuits conventionnels et peu reconnus par les services de conseil et de développement. Plusieurs perspectives pourraient être explorées pour poursuivre cette recherche. En effet, lors de nos enquêtes, nous n'avons pas rencontré de PAF dans certaines zones: nord de Grande-Terre et nord de BasseTerre, ce qui peut signifier sa faible représentation, voire son absence dans ces zones. Mais cela peut aussi relever d'un biais lié à la base de données initiale. Il est alors possible que cette PAF soit encore plus diverse que les cinq types identifiés et qu'il existe des types présentant des synergies entre l'ensemble des indicateurs considérés dans cette étude. Plus d'indicateurs pourraient également être considérés, en particulier ceux relatifs aux caractéristiques agroécologiques 
du système. Par exemple, l'indicateur de biodiversité pourrait non seulement considérer la biodiversité planifiée, mais aussi la biodiversité «associée» de l'exploitation (faune et flore colonisant l'agroécosystème), qui joue elle aussi un rôle dans la fourniture de services écosystémiques et les avantages que peuvent en tirer les agriculteurs (Altieri, 1999). Des indicateurs supplémentaires relatifs aux services écosystémiques pourraient également être pris en compte pour mieux analyser le lien entre pratiques, services écosystémiques et avantages. Dans le contexte spécifique de la PAF de Guadeloupe, il pourrait s'agir en particulier de services écosystémiques de régulation des bioagresseurs, de l'eau ou du climat. Il paraît aussi important de mieux caractériser la part des revenus extra-agricoles dans ces systèmes, puisque la pluriactivité constitue une caractéristique clé facilitant dans certains cas la prise de risque au sein de l'exploitation ou au contraire déterminant la mise en place de systèmes de culture et d'élevage peu exigeants en maind'œuvre.

\section{Conclusion}

Cette étude, combinant enquêtes, typologie et évaluation des avantages tirés par les agriculteurs des pratiques agroécologiques, permet de caractériser une PAF souvent informelle et peu présente au sein des circuits d'aide en Guadeloupe, et d'analyser le rôle joué par les pratiques agroécologiques dans les performances des exploitations. Elle montre que cette PAF est diverse et que l'hybridation faite entre pratiques conventionnelles et agroécologiques dépend de l'atelier de production dominant. En effet, ce dernier influe sur le nombre de pratiques agroécologiques et sur les performances mesurées en termes de couverture des besoins caloriques, de diminution des charges intermédiaires ou de génération de valeur ajoutée commerciale. Néanmoins, comparés entre eux, aucun des types n'obtient de performances élevées sur l'ensemble des indicateurs mesurés. L'analyse de la façon d'améliorer les synergies entre indicateurs permet d'identifier des leviers pour favoriser la transition agroécologique de cette PAF.

Plusieurs pistes ont aussi été identifiées pour améliorer l'outil construit. Cet outil sera utilisé avec les agriculteurs pour valider avec eux les possibles leviers à actionner à l'échelle de l'exploitation pour s'engager dans cette transition agroécologique.

Remerciements. Cette recherche a été réalisée dans le cadre du projet AgroEcoDiv, financé par PO FEDER 2014-2020. Nous remercions les agriculteurs qui ont accepté de participer à cette recherche. Nous remercions également les relecteurs anonymes qui ont contribué à améliorer le document.

\section{Références}

Altieri MA. 1999. The ecological role of biodiversity in agroecosystems. Agriculture, Ecosystems and Environment 74: 19-31.
Altieri MA. 2002. Agroecology: the science of natural resource management for poor farmers in marginal environment. Agriculture, Ecosystems and Environment 93: 1-24.

Bezombes H, Cardon A, Hostache C, Kempen C, Manceaux J, Mars C, et al. 2015. Étude des mécanismes d'attribution des aides publiques agricoles et analyses des stratégies et des enjeux de la petite agriculture familiale guadeloupéenne, vis-à-vis du système de subventions agricoles, dans le cadre de la réforme de la politique agricole commune 2015-2020 et de la réforme législative du Code rural de l'agriculture et de la pêche, par l'approbation de la loi d'Avenir le 13 octobre 2014. Rapport de Mission Jeune expert. Angers: Istom, $189 \mathrm{p}$.

Burgess 2005. Guide nutrition famille. Rome: Food and Agriculture Organization of the United Nations, $134 \mathrm{p}$.

CICES, 2013. Common International Classification of Ecosystem Services. Available from http://cices.eu [consulté le 12/07/ 2017].

Darnhofer I, Lamine C, Strauss A, Navarrete M. 2016. The resilience of family farms: towards a relational approach. Journal of Rural Studies 44: 111-122.

Duru M, Fares M, Therond O. 2014. Un cadre conceptuel pour penser maintenant (et organiser demain) la transition agroécologique de l'agriculture dans les territoires. Cahiers Agricultures 23: 84-95. DOI: 10.1684/agr.2014.0691.

Godinot O. 2014. Proposition de nouveaux indicateurs d'efficience d'utilisation de l'azote à l'échelle du système de production agricole et du territoire. Thèse Rennes Agrocampus Ouest. p. 135 .

Graeub BE, Chappell MJ, Wittman H, Ledermann S, Bezner Kerr R, Gemmill-Herren B. 2016. The state of family farms in the world. World Development 87: 1-15.

Inra. 2015. Atelier Trans'Act: vers une performance socio économique dans une logique agroécologique. Actes-Séminaire $1^{\text {er }}$ au 5 février 2015, p. 129.

Laws S, Harper C, Jones N, Marcus R. 2013. Research for development: a practical guide. USA: Sage Publications.

Le Gal PY, Bernard J, Moulin CH. 2013. Supporting strategic thinking of smallholder dairy farmers using a whole farm simulation tool. Trop Anim Health Pro 45: 1119-1129.

Lowder SK, Skoet J, Raney T. 2016. The number, size, and distribution of farms, smallholder farms, and family farms worldwide. World Development 87: 16-29.

Météo France. 2017. Hauteurs de pluies annuelles (mm) en 2017. Écarts aux normales 1981-2010. Carte annuelle des pluies en Guadeloupe. pdf. p.1.

Mantran M, Lucien-Brun M, Diman J-L. 2016. Productive dynamics and agroecological potential of the territory of Guadeloupe: a geographer analysis. Proceedings of the 52nd Annual Meeting "Engineering ecological modernization of agriculture; Exploring the potential of tropical biological resources for innovation; Towards a bio-economic development of Caribbean countries". Isabela, PRI: Caribbean Food Crops Society, p. 1.

Power AG. 2010. Ecosystem services and agriculture: tradeoffs and synergies. Philosophical Transactions of the Royal Society of London B: Biological Sciences 365: 2959-2971. DOI: 10.1098/ rstb.2010.0143.

Semjen I. 2015. Les pratiques d'intégration agriculture-élevage au sein des exploitations guadeloupéennes. Mémoire. Angers : Istom, $126 \mathrm{p}$. 
Semjen I. 2017. Relecture de l'évolution d'un système agraire antillais: le cas de la Guadeloupe. Support de cours agrodéveloppement. Angers: Istom. p. 101.

Sempore AW, Andrieu N, Nacro HB, Sedogo MP, Le Gal PY. 2015. Relevancy and role of whole-farm models in supporting smallholder farmers in planning their agricultural season. Environmental Modelling and Software 68: 147-155. DOI: 10.1016/j.envsoft.2015.02.015.
Sourisseau JM, ed. 2015. Family farming and the worlds to come. Dordrecht (Pays-Bas): Springer; Versailles (France): Quae, 371 p. DOI: 10.1007/978-94-017-9358-2.

Stark FS, Fanchone A, Semjen I, Moulin CH, Archimede H. 2016. Crop-livestock integration, from single practice to global functioning in the tropics: case studies in Guadeloupe. European Journal of Agronomy 80: 9-20. DOI: 10.1016/j. eja.2016.06.004.

Citation de l'article : Rasse C, Andrieu N, Diman J-L, Fanchone A, Chia E. 2018. Utilisation de pratiques agroécologiques et performances de la petite agriculture familiale: le cas de la Guadeloupe. Cah. Agric. 27: 55002. 